\title{
Antimicrobial Resistance, Virulence genes, and Phylogenetic Characteristics of pathogenic Escherichia coli isolated from Patients and Swine suffering from Diarrhea
}

\section{Kyung-Hyo Do}

Chungbuk National University

\section{Kwang-Won Seo}

Korea Zoonosis Research Institute, Jeonbuk National University

Wan-Kyu Lee ( $\square$ wklee@cbu.ac.kr)

Chungbuk National University

\section{Research Article}

Keywords: Swine, Patients, Diarrhea, Escherichia coli, Virulence factors, Antimicrobial resistance, multilocus sequence typing

Posted Date: April 7th, 2022

DOI: https://doi.org/10.21203/rs.3.rs-1394995/v3

License: (c) (1) This work is licensed under a Creative Commons Attribution 4.0 International License.

Read Full License 


\section{Abstract}

Background: Escherichia (E.) coli causes colibacillosis in swine and humans, and is frequently associated with antimicrobial resistance. In this study we aimed to compare antimicrobial resistance, O-serogroups, virulence genes, and multi-locus sequence type of $E$. coli between isolates from pigs and patients suffering from diarrhea, and the most prevalent pathogenic $E$. coli strain from swine isolates in Korea.

Methods: We tested 64 and 50 E. coli strains from pigs and patients suffering from diarrhea for antimicrobial susceptibility test, virulence genes, 0-serogroups, and multi-locus sequence typing.

Results: We confirmed that isolates from swine showed significantly higher resistance than from those from patients, especially to fluoroquinolone (ciprofloxacin: $37.5 \%$ and $10.0 \%$; norfloxacin: $29.7 \%$ and $8.0 \%$, respectively). Stx 1 (23 isolates, $46.0 \%$ ) was most frequently detected in patients followed by stx2 (19 isolates, 38.0\%). There was no significant difference in stx2 (swine: $23.4 \%$, patients: $38.0 \%$ ). In isolates from patients, 0157 (12.0\%) was the most prevalent O-serogroup, and two isolates (3.1\%) from pigs were confirmed to have 0157. Additionally, sequence type (ST) 10 (swine: 6 isolates, patients: 2 isolates) and ST 88 (swine: 2 isolates, patients: 1 isolate) were simultaneously detected.

Conclusions: We confirmed that isolates from both pigs and patients had similar virulence traits and they were phylogenetically similar. Moreover, antimicrobial resistance was significantly higher in pigs than in patients. These results suggest that there are risks for cross-infection and transfer of antimicrobial resistance between pigs and human.

\section{Background}

Escherichia coli (E. coli) causes colibacillosis, a common disease that occurs in pigs and humans (1). Colibacillosis in pigs has a significant economic impact on the pig farming due to its association with high rates of morbidity and mortality (2). Moreover, pathogenic $E$. coli causes diarrhea and hemorrhagic colitis in humans, with life-threatening complications, such as hemolytic uremic syndrome (3).

Antimicrobial agents are frequently used for the treatment of colibacillosis (4). In Korea, the largest number of antimicrobials was sold for use in pigs (55\%, 507 tons), which was higher than that sold for use in poultry (17\%, 155 tons) and cattle (11\%, 99 tons) (5). Consequently, antimicrobial resistance was much higher in swine isolates than that in isolates of other livestock (5). Since antimicrobial resistance can be transferred from pigs to humans, there is a need for the surveillance of antimicrobial resistance in pigs (6).

The pathogenicity of $E$. coli is determined by virulence genes (toxin and adhesin) and/or O-serogroups (7). The frequency of these virulence factors is known to vary over time and based on the host. Although a variety of 0 -serogroups have been associated with colibacillosis, a limited number of serogroups have been reported for specific disease, such as postweaning diarrhea, edema disease, and hemorrhagic colitis $(8,9)$. Those virulence genes were frequently detected in isolates from both pigs and humans. Pigs are 
considered the primary reservoirs of pathogenic E. coli in human infections and food products, such as pork products, and thus are the carries for the transmission of pathogenic E. coli (2). Therefore, it is important to establish the clonal relationship between strains from different hosts and diseases to assess the risk of zoonotic infections (10).

Although there have been many studies that focused on the antimicrobial resistance of $E$. coli, but most studies analyzed the antimicrobial resistance of commensal $E$. coli. There have been fewer studies on the correlation between antimicrobial resistance and virulence factors of $E$. coli in diarrheic pigs and in patients suffering from diarrhea. In this study, we aimed to compare antimicrobial resistance, 0 serogroups, virulence genes, and multi-locus sequence type (MLST) of most dominant pathogenic E. coli from pigs with the isolates obtained from patients suffering from diarrhea in Korea.

\section{Methods}

\section{E. coli strains}

For this study, the most dominant pathogenic E. coli strain from pigs suffering from edema disease and postweaning diarrhea in Korea were used, according to a previous study (9). Twenty-nine strains of enterotoxigenic E. coli (ETEC), 28 strains of shiga toxin-producing E. coli (STEC), and seven strains of ETEC/STEC were selected. For isolating these strains, aseptically collected intestinal and fecal samples were inoculated onto MacConkey agar (Becton Dickinson, MD, USA). After overnight incubation at $37^{\circ} \mathrm{C}$, only pure cultured colonies that were pink in color were selected and transferred to blood agar (Asan Pharmaceutical, Korea). Suspected colonies were identified as $E$. coli by using the VITEK $\otimes$ system (bioMéreiux, Marcy l'Etoile, France). The tested isolates were stored in $50 \%$ glycerol stock at $-70{ }^{\circ} \mathrm{C}$ until further characterization. Fifty E. coli strains were isolated from patients suffering from diarrhea and kindly provided by the National Culture Collection for Pathogens (NCCP), Korea.

\section{Detection Of Virulence Gene And 0-serogroups}

Reference E. coli strains provided by the Animal and Plant Quarantine Agency (Korea) were used as positive controls for polymerase chain reaction (PCR) Aanalysis, they included: 7805 (F4:LT:STa:STb:EAST-I:paa), 6611 (stx1:stx2:eae:EAST-I:paa), 1033 (F18:AIDA-I), 2316 (F6:STa:STb:EASTI:paa), and 13316 (F5:F41:STa:paa); and 3463 was used as a negative control. Template DNA for PCR analysis was extracted using the boiling method. PCR test described below was conducted for analyzing F4, F5, F6, F18, F41, eae, paa, AIDA-I, stx1, stx2, aggR, ipaH, LT, ST, and EAST-I as previously described (9). The reaction volume $(20 \mu \mathrm{L})$ was composed of $2 \times$ EmeraldAmp Master Mix (TaKaRa, Japan), $2 \mu \mathrm{M}$ of each primer, and $3 \mu \mathrm{L}$ of DNA template. TaKaRa PCR Thermal Cycler Dice Gradient TP600 (TaKaRa, Japan) was used for performing the PCR analysis. After amplification, the resultant products underwent electrophoresis on 2\% agarose gel using Mupid-exu AD140 (TaKaRa, Japan), stained with BlueMango (BioD, Korea), and was confirmed using BluePad (BioD, Korea). O-serogroup typing was performed using 
the slide agglutination technique in the Animal and Plant Quarantine Agency (Korea) using rabbit antisera purchased from Serum Staten Institute (Denmark).

\section{Antimicrobial Susceptibility Test}

The following 21 antimicrobial agents were selected by referring to the Clinical and Laboratory Standards Institute (CLSI) guidelines and were used in this study: gentamicin; streptomycin; neomycin, kanamycin, amikacin, amoxicillin / clavulanate, cephalothin, cefazolin, cefoxitin, cefepime, nalidixic acid, ciprofloxacin, norfloxacin, tetracycline, doxycycline, ampicillin, trimethoprim / sulfamethoxazole, chloramphenicol, colistin, and clindamycin (11). Each antimicrobial disc was purchased from BectonDickinson (BD, USA). Antimicrobial susceptibility testing was carried out using the Kirby Bauer disk diffusion method (12). Strains resistant to three or more CLSI subclass drugs according to the Magiorakos criteria were considered as multidrug resistant strains (13).

\section{Multi-locus Sequence Typing (Mlst)}

All processes, including genomic DNA extraction, PCR amplification, Sanger sequencing, and assembly were performed by Macrogen (Macrogen, South Korea). Genomic DNA were extracted using a Bio-Rad (USA). MLST was performed using partial sequences of seven house-keeping genes (adk, fumC, gyrB, icd, $m d h$, purA and recA), as previously described. PCR was performed with $20 \mathrm{ng}$ of genomic DNA as template in a $30 \mu \mathrm{L}$ reaction mixture, using Dr. MAX DNA Polymerase (Doctor protein INC, South Korea) as follows: activation of Taq polymerase at $95^{\circ} \mathrm{C}$ for $5 \mathrm{~min} ; 35$ cycles at $95^{\circ} \mathrm{C}$ for $30 \mathrm{sec}, 52^{\circ} \mathrm{C}$ for 30 sec, and $72{ }^{\circ} \mathrm{C}$ for $1 \mathrm{~min}$; and a final $10 \mathrm{~min}$ step at $72{ }^{\circ} \mathrm{C}$. The products obtained after amplification were purified using a multiscreen filter plate (Millipore Corp. USA). Sequencing was performed using a PRISM BigDye Terminator v3.1 Cycle Sequencing Kit. The Mixture was incubated at $95^{\circ} \mathrm{C}$ for $5 \mathrm{~min}$, followed by 5 min on ice and then analyzed in an ABI PRISM 3730XL DNA analyzer (Applied Biosystems, USA).

Sequence types (ST) were assigned online (http://pubmlst.org/biqsdb?

$\mathrm{db}=$ pubmlst_ecoli_achtman_seqdef).

\section{Statistical analysis}

Statistical analysis was performed using the SPSS version 12.0 program (SPSS, Chicago, Illinois, USA). Chi-squared test was performed to analyze the pathogenic characteristics and the rate of antimicrobial resistance of $E$. coli from diarrheic pigs and patients.

\section{Results}

\section{Antimicrobial susceptibility test}


Table 1 describes the results of the antimicrobial susceptibility test. The isolates from pigs showed significantly higher resistance to gentamicin (51.6\%), kanamycin (85.9\%), amikacin (71.9\%), nalidixic acid (65.6\%), ciprofloxacin (37.5\%), norfloxacin (29.7\%), tetracycline (82.8\%), doxycycline (76.6\%), trimethoprim / sulfamethoxazole (51.6\%), and chloramphenicol (79.7\%) as compared to those from patients (gentamicin: $10.0 \%$, kanamycin: $30.0 \%$, amikacin: $2.0 \%$, nalidixic acid: $42.0 \%$, ciprofloxacin: 10.0\%, norfloxacin: $8.0 \%$, tetracycline: $40.0 \%$, doxycycline: $22.0 \%$, trimethoprim / sulfamethoxazole: $26.0 \%$, and chloramphenicol: $28.0 \%$ ). Isolates from patients showed significantly higher resistance to amoxicillin / clavulanic acid (66.0\%) than those from pigs (15.6\%). The resistance to cefepime, which is the 4th generation of cephalosporins, was not detected in all isolates. 
Table 1

Antimicrobial resistance of Escherichia colifrom diarrheic pigs and patients.

\begin{tabular}{|c|c|c|c|c|}
\hline \multirow[t]{3}{*}{ Antimicrobial agents $\left.{ }^{1}\right)$} & & \multicolumn{3}{|c|}{ No. (\%) of resistant isolates } \\
\hline & & Swine & Patients & Total \\
\hline & & $(n=64)$ & $(n=50)$ & $(n=114)$ \\
\hline \multirow[t]{5}{*}{ Aminoglycosides } & GM & $33(51.6 \%)$ & $5(10.0 \%)$ & $38(33.3 \%)^{* *}$ \\
\hline & $S$ & $41(64.1 \%)$ & $30(60.0 \%)$ & $71(62.3 \%)$ \\
\hline & $\mathbf{N}$ & $29(45.3 \%)$ & $11(22.0 \%)$ & $40(35.1 \%)^{*}$ \\
\hline & $\mathrm{K}$ & $55(85.9 \%)$ & $15(30.0 \%)$ & $70(61.4 \%)^{\star \star}$ \\
\hline & AN & $46(71.9 \%)$ & $1(2.0 \%)$ & $47(41.2 \%)^{\star \star}$ \\
\hline$\beta$-lactam/lactamase inhibitor & AMC & $10(15.6 \%)$ & $33(66.0 \%)$ & $43(37.7 \%)^{\star \star}$ \\
\hline \multirow[t]{2}{*}{ Cephalosporin 1} & CF & 14 (21.9\%) & 15 (30.0\%) & 29 (25.4\%) \\
\hline & $\mathrm{CZ}$ & $8(12.5 \%)$ & $5(10.0 \%)$ & $13(11.4 \%)$ \\
\hline Cephalosporin 2 & FOX & $5(7.8 \%)$ & $3(6.0 \%)$ & $8(7.0 \%)$ \\
\hline Cephalosporin 4 & FEP & $0(0.0 \%)$ & $0(0.0 \%)$ & $0(0.0 \%)$ \\
\hline Quinolone & NA & $42(65.6 \%)$ & $21(42.0 \%)$ & $63(55.3 \%)^{*}$ \\
\hline \multirow[t]{2}{*}{ Fluoroquinolone } & CIP & $24(37.5 \%)$ & $5(10.0 \%)$ & $29(25.4 \%)^{\star \star}$ \\
\hline & NOR & $19(29.7 \%)$ & $4(8.0 \%)$ & $23(20.2 \%)^{\star \star}$ \\
\hline \multirow[t]{2}{*}{ Tetracyclines } & TE & 53 (82.8\%) & 20 (40.0\%) & $73(64.0 \%)^{\star *}$ \\
\hline & DOX & $49(76.6 \%)$ & $11(22.0 \%)$ & $60(52.6 \%)^{\star \star}$ \\
\hline Aminopenicillin & AMP & $53(82.8 \%)$ & $36(72.0 \%)$ & $89(78.1 \%)$ \\
\hline Folate pathway inhibitors & SXT & $33(51.6 \%)$ & $13(26.0 \%)$ & $46(40.4 \%)^{\star \star}$ \\
\hline Phenicols & C & $51(79.7 \%)$ & $14(28.0 \%)$ & $65(57.0 \%)^{\star \star}$ \\
\hline
\end{tabular}

1) GM: gentamicin; S: streptomycin; N: neomycin; CF: cephalothin; CZ: cefazolin; FEP: cefepime; FOX: cefoxitin; NA: nalidixic acid; CIP: ciprofloxacin; NOR: norfloxacin; AMP: ampicillin; AMC: amoxicillin / clavulanic acid, SXT: trimethoprim / sulfamethoxazole; C: chloramphenicol; CL: colistin; TE: tetracycline

${ }^{*}$ Significant difference between origin of isolates $(p<0.05)$.

${ }^{* *}$ Significant difference between origin of isolates $(p<0.01)$. 


\begin{tabular}{|c|c|c|c|c|}
\hline \multirow[t]{3}{*}{ Antimicrobial agents ${ }^{1)}$} & & \multicolumn{3}{|c|}{ No. (\%) of resistant isolates } \\
\hline & & Swine & Patients & Total \\
\hline & & $(n=64)$ & $(n=50)$ & $(n=114)$ \\
\hline Polymyxins & $\mathrm{CL}$ & $1(1.6 \%)$ & $0(0.0 \%)$ & $1(0.9 \%)$ \\
\hline Lincosamide & $\mathrm{CC}$ & $64(100.0 \%)$ & $50(100.0 \%)$ & $114(100.0 \%)$ \\
\hline \multicolumn{5}{|c|}{$\begin{array}{l}\text { 1) GM: gentamicin; S: streptomycin; N: neomycin; CF: cephalothin; CZ: cefazolin; FEP: cefepime; FOX } \\
\text { cefoxitin; NA: nalidixic acid; CIP: ciprofloxacin; NOR: norfloxacin; AMP: ampicillin; AMC: amoxicillin / } \\
\text { clavulanic acid, SXT: trimethoprim / sulfamethoxazole; C: chloramphenicol; CL: colistin; TE: } \\
\text { tetracycline }\end{array}$} \\
\hline \multicolumn{5}{|c|}{${ }^{*}$ Significant difference between origin of isolates $(p<0.05)$. } \\
\hline
\end{tabular}

\section{Multidrug Resistance Rates}

The results of multidrug resistance analysis are shown in Table 2 . We found that $23.4 \%$ of pig isolates were resistant to seven subclasses ( 15 isolates), which were the most prevalent, while $18.0 \%$ (9 isolates) of isolates of patients showed resistant to four subclasses. Especially, only pig isolates showed resistance to 10 subclasses (4 isolates, 6.3\%), while no isolates from patients showed resistance to 10 subclasses. In terms of multidrug resistance in those resistant to three or more subclasses of drugs among the 14 subclasses of drugs tested, $93.8 \%$ (60 isolates) of pig isolates and $86.0 \%$ (43 isolates) of patient isolates showed multidrug resistance. 
Table 2

Multidrug resistance of $E$. coli from diarrheic pigs and patients in Korea.

\begin{tabular}{|llll|}
\hline Resistance Patterns ${ }^{1)}$ & $\begin{array}{l}\text { Swine } \\
(\mathbf{n = 6 4 )}\end{array}$ & $\begin{array}{l}\text { Patients } \\
(\mathbf{n = 5 0 )}\end{array}$ & $\begin{array}{l}\text { Total } \\
(\mathbf{n}=114)\end{array}$ \\
\hline $\mathbf{0}$ subclass & $0(0.0 \%)$ & $0(0.0 \%)$ & $0(0.0 \%)$ \\
\hline $\mathbf{1}$ subclass & $3(4.7 \%)$ & $0(0.0 \%)$ & $3(2.6 \%)$ \\
\hline $\mathbf{2}$ subclasses & $1(1.6 \%)$ & $7(14.0 \%)$ & $8(7.0 \%)^{*}$ \\
\hline $\mathbf{3}$ subclasses & $0(0.0 \%)$ & $7(14.0 \%)$ & $7(6.1 \%)^{\star \star}$ \\
\hline $\mathbf{4}$ subclasses & $7(10.9 \%)$ & $9(18.0 \%)$ & $16(14.0 \%)$ \\
\hline $\mathbf{5}$ subclasses & $11(17.2 \%)$ & $8(16.0 \%)$ & $19(16.7 \%)$ \\
\hline $\mathbf{6}$ subclasses & $13(20.3 \%)$ & $8(16.0 \%)$ & $21(18.4 \%)$ \\
\hline 7 subclasses & $15(23.4 \%)$ & $6(12.0 \%)$ & $21(18.4 \%)$ \\
\hline $\mathbf{8}$ subclasses & $6(9.4 \%)$ & $3(6.0 \%)$ & $9(7.9 \%)$ \\
\hline 9 subclasses & $4(6.3 \%)$ & $2(4.0 \%)$ & $6(5.3 \%)$ \\
\hline 10 subclasses & $4(6.3 \%)$ & $0(0.0 \%)$ & $4(3.5 \%)$ \\
\hline Multi-Drug Resistance & $6(93.8 \%)$ & $43(86.0 \%)$ & $103(90.4 \%)$ \\
\hline 1$)$ Antimicrobial subclasses defined by the Clinical and Laboratory Standards Institute (CLSI) are \\
\hline used.
\end{tabular}

\section{Virulence Factors}

The prevalence of virulence genes (Table 3 ) and pathotypes with combination of virulence genes (virotype) of E. coli from diarrheic pigs and patients were compared (Table 4). The most prevalent virulence genes in pigs were F18 (35 isolates, $54.7 \%$ ) and stx2e (35 isolates, $54.7 \%)$. While stx 1 (23 isolates, $46.0 \%$ ) was most frequently detected in patients, followed by stx2 (19 isolates, $38.0 \%)$. There was no significant difference in the prevalence of stx2 (swine: $23.4 \%$, patients: $38.0 \%$ ).

ETEC ( 29 isolates, $45.3 \%$ ) was the most prevalent pathotype in pigs, followed by STEC ( 28 isolates, $43.8 \%$ ). Similarly, most isolates (27 isolates, $54.0 \%$ ) from patients were confirmed to have STEC, which was the most prevalent pathotype, followed by ETEC (11 isolates, 22.0\%). In contrast to swine isolates, there were various pathotypes in patients, such as enteroaggregative E. coli (EAEC) (3 isolates, 6.0\%), 
enteroinvasive E. coli (EIEC) (1 isolate, 2.0\%), enteropathogenic E. coli (EPEC) (1 isolate, 2.0\%), STEC/EPEC (3 isolates, 6.0\%), and STEC/EAEC (2 isolates, 4.0\%). In STEC, 17 isolates from patients encoded stx2 gene and 10 isolates encoded stx 1 gene; stx 1 gene was not found in swine isolates. In ETEC, EAST-I gene was only detected in isolates from swine, and both LT and ST toxin genes were detected in isolates from swine and patients.

Table 3

Virulence genes of $E$. coli from diarrheic pigs and patients in Korea.

\begin{tabular}{|c|c|c|c|}
\hline \multirow[t]{2}{*}{ Viriulence genes } & \multicolumn{3}{|c|}{ No. (\%) of pathogenic $E$. coli isolates } \\
\hline & $\begin{array}{l}\text { Swine } \\
(n=64)\end{array}$ & $\begin{array}{l}\text { Patients } \\
(n=50)\end{array}$ & $\begin{array}{l}\text { Total } \\
(n=114)\end{array}$ \\
\hline F4 & $29(45.3 \%)$ & $0(0.0 \%)$ & $\left.29(25.4 \%)^{\star \star 1}\right)$ \\
\hline F18 & $35(54.7 \%)$ & $0(0.0 \%)$ & $35(30.7 \%)^{\star \star}$ \\
\hline eae & $0(0.0 \%)$ & $4(8.0 \%)$ & $4(3.5 \%)^{\star}$ \\
\hline AIDA-I & $17(26.6 \%)$ & $0(0.0 \%)$ & $17(14.9 \%)^{\star \star}$ \\
\hline $\operatorname{aggR}$ & $0(0.0 \%)$ & $3(6.0 \%)$ & $3(2.6 \%)$ \\
\hline ipaH & $0(0.0 \%)$ & $1(2.0 \%)$ & $1(0.9 \%)$ \\
\hline LT & $29(45.3 \%)$ & $6(12.0 \%)$ & $35(30.7 \%)^{* \star}$ \\
\hline ST & $29(45.3 \%)$ & $8(16.0 \%)$ & $37(32.5 \%)^{\star *}$ \\
\hline EAST-I & $36(56.3 \%)$ & $0(0.0 \%)$ & $36(31.6 \%)^{* *}$ \\
\hline stx1 & $0(0.0 \%)$ & $23(46.0 \%)$ & $23(20.2 \%)^{\star *}$ \\
\hline stx2 & $15(23.4 \%)$ & $19(38.0 \%)$ & $34(29.8 \%)$ \\
\hline$s t \times 2 e$ & $35(54.7 \%)$ & $0(0.0 \%)$ & $35(30.7 \%)^{\star *}$ \\
\hline \multicolumn{4}{|c|}{ 1) * Significant difference between origin of isolates $(p<0.05)$. } \\
\hline${ }^{* *} \mathrm{~S}$ & & . & $s(p<0.01)$ \\
\hline
\end{tabular}


Table 4

Comparison of pathotypes of 114 Escherichia coli from diarrheic pigs and patients in Korea

\begin{tabular}{|c|c|c|c|}
\hline \multirow{2}{*}{$\begin{array}{l}\text { Pathotype } \\
\text { Virotype }\end{array}$} & \multicolumn{3}{|c|}{ No. (\%) of pathogenic E. coli isolates } \\
\hline & $\begin{array}{l}\text { Swine } \\
(n=64)\end{array}$ & $\begin{array}{l}\text { Patients } \\
(n=50)\end{array}$ & $\begin{array}{l}\text { Total } \\
(n=114)\end{array}$ \\
\hline ETEC & 29 (45.3\%) & $11(22.0 \%)$ & $\left.40(35.1 \%)^{* 1}\right)$ \\
\hline F4:LT:ST:EAST-I & $29(100.0 \%)$ & - & $29(72.5 \%)$ \\
\hline ST & - & $5(45.5 \%)$ & $5(12.5 \%)$ \\
\hline LT & - & $3(27.3 \%)$ & $3(7.5 \%)$ \\
\hline LT:ST & - & $3(27.3 \%)$ & $3(7.5 \%)$ \\
\hline STEC & $28(43.8 \%)$ & $27(54.0 \%)$ & $55(48.2 \%)$ \\
\hline F18:AIDA-l:stx2e & $14(50.0 \%)$ & - & $14(25.0 \%)$ \\
\hline stx1 & - & $10(37.0 \%)$ & $10(17.9 \%)$ \\
\hline stx1:stx2 & - & $10(37.0 \%)$ & $10(17.9 \%)$ \\
\hline F18:stx2:stx2e & $7(25.0 \%)$ & - & $7(12.5 \%)$ \\
\hline stx2 & - & $7(25.9 \%)$ & $7(12.5 \%)$ \\
\hline F18:stx2e & $4(14.3 \%)$ & - & $4(7.1 \%)$ \\
\hline F18:AIDA-I:stx2:stx2e & $3(10.7 \%)$ & - & $3(5.4 \%)$ \\
\hline EAEC & $0(0.0 \%)$ & $3(6.0 \%)$ & $3(2.6 \%)$ \\
\hline $\operatorname{aggR}$ & - & $3(100.0 \%)$ & $3(100.0 \%)$ \\
\hline EIEC & $0(0.0 \%)$ & $1(2.0 \%)$ & $1(0.9 \%)$ \\
\hline ipaH & - & $1(100.0 \%)$ & $1(100.0 \%)$ \\
\hline EPEC & $0(0.0 \%)$ & $1(2.0 \%)$ & $1(0.9 \%)$ \\
\hline eae & - & $1(100.0 \%)$ & $1(100.0 \%)$ \\
\hline ETEC/STEC & 7 (10.9\%) & $0(0.0 \%)$ & $7(6.1 \%)^{*}$ \\
\hline F18:stx2:stx2e:EAST-I & $5(71.4 \%)$ & - & $5(71.4 \%)$ \\
\hline F18:stx2e:EAST-I & $2(28.6 \%)$ & - & $2(28.6 \%)$ \\
\hline STEC/EPEC & $0(0.0 \%)$ & $3(6.0 \%)$ & $3(2.6 \%)$ \\
\hline
\end{tabular}




\begin{tabular}{|llll|}
\hline Pathotype & \multicolumn{3}{l|}{ No. (\%) of pathogenic E. coli isolates } \\
\cline { 2 - 4 } Virotype & $\begin{array}{l}\text { Swine } \\
(\mathbf{n}=64)\end{array}$ & $\begin{array}{l}\text { Patients } \\
(\mathbf{n}=\mathbf{5 0})\end{array}$ & $\begin{array}{l}\text { Total } \\
(\mathbf{n}=114)\end{array}$ \\
\hline stx1:eae & - & $3(100.0 \%)$ & $3(100.0 \%)$ \\
\hline STEC/EAEC & $0(0.0 \%)$ & $2(4.0 \%)$ & $2(1.8 \%)$ \\
\hline stx2:aggR & - & $2(100.0 \%)$ & $2(100.0 \%)$ \\
\hline Non-detected & $0(0.0 \%)$ & $2(4.0 \%)$ & $2(1.8 \%)$ \\
\hline 1) ${ }^{*}$ Significant difference between origin of isolates $(p<0.05)$. \\
\hline
\end{tabular}

\section{O-serogroups And Virotype}

The 0-serogroups and virotypes is presented in Table 5. There was no 0149 and 0139 isolates from patients while most isolates from swine were confirmed in 0149 (28 isolates, 43.8\%) and 0139 (13 isolates, 20.3\%). In isolates from patients, 0157 ( 6 isolates, 12.0\%) was the most prevalent 0-serogroup, and also 2 isolates (3.1\%) of swine was confirmed in 0157. Interestingly, 0157 isolates from swine were ETEC (F4:LT:STb:EAST-I) which is associated with diarrhea, while all isolates from patients were confirmed in STEC (stx1, stx2, and stx1:stx2) which is associated with hemorrhagic colitis. 
Table 5

Comparison of O-serogroups and virotypes of 114 Escherichia coli isolates from diarrheic pigs and patients in Korea

\begin{tabular}{|c|c|c|c|}
\hline \multirow{2}{*}{$\begin{array}{l}\text { O-serogroup } \\
\text { Virotype }\end{array}$} & \multicolumn{3}{|c|}{ No. (\%) of pathogenic E. coli isolates } \\
\hline & Swine $(n=64)$ & Patients $(n=50)$ & Total $(n=114)$ \\
\hline 0149 & $28(43.8 \%)$ & $0(0.0 \%)$ & $\left.28(24.6 \%)^{\star \star 1}\right)$ \\
\hline F4:LT:STb:EAST-I & 20 & - & 20 \\
\hline F18:Stx2e & 3 & - & 3 \\
\hline F18:AIDA-I:Stx2:Stx2e & 2 & - & 2 \\
\hline F18:Stx2:Stx2e & 2 & - & 2 \\
\hline F18:AIDA-I:Stx2e & 1 & - & 1 \\
\hline 0139 & $13(20.3 \%)$ & $0(0.0 \%)$ & $13(11.4 \%)^{* *}$ \\
\hline F18:AIDA-I:Stx2e & 13 & - & 13 \\
\hline 0157 & $2(3.1 \%)$ & $6(12.0 \%)$ & $8(7.0 \%)$ \\
\hline F4:LT:STb:EAST1 & 2 & - & 2 \\
\hline stx1:stx2 & - & 3 & 3 \\
\hline stx2 & - & 2 & 2 \\
\hline stx1 & - & 1 & 1 \\
\hline 026 & $0(0.0 \%)$ & 4 (8.0\%) & $4(3.5 \%)^{*}$ \\
\hline stx1:stx2 & - & 2 & 2 \\
\hline stx1 & - & 1 & 1 \\
\hline stx1:eaeA & - & 1 & 1 \\
\hline 025 & $0(0.0 \%)$ & $4(8.0 \%)$ & $4(3.5 \%)^{*}$ \\
\hline
\end{tabular}

1) * Significant difference between origin of isolates $(p<0.05)$.

${ }^{*}$ Significant difference between origin of isolates $(p<0.01)$.

2) O_UT: Untypeable

3) Non-virulence gene detected

4) Other serogroup (number of isolates): 06 (3), 018 (3), 0100 (3), 0136 (3), 011 (2), 021 (2), 055 (2), 0146 (2), 015 (1), 028 (1), 057 (1), 078 (1), 091 (1), 098 (1), 0103 (1), 0111 (1), 0121 (1), 0145 (1), $0174(1)$. 


\begin{tabular}{|c|c|c|c|}
\hline \multirow{2}{*}{$\begin{array}{l}\text { O-serogroup } \\
\text { Virotype }\end{array}$} & \multicolumn{3}{|c|}{ No. (\%) of pathogenic E. coli isolates } \\
\hline & Swine $(n=64)$ & Patients $(n=50)$ & Total $(n=114)$ \\
\hline LT & - & 3 & 3 \\
\hline stx1 & - & 1 & 1 \\
\hline 0104 & $0(0.0 \%)$ & $4(8.0 \%)$ & $4(3.5 \%)^{*}$ \\
\hline stx2:aggR & - & 2 & 2 \\
\hline stx1 & - & 1 & 1 \\
\hline stx2 & - & 1 & 1 \\
\hline 0159 & $0(0.0 \%)$ & $4(8.0 \%)$ & $4(3.5 \%)^{*}$ \\
\hline ST & - & 3 & 3 \\
\hline stx1:stx2 & - & 1 & 1 \\
\hline O_UT'2) & $7(10.9 \%)$ & $11(22.0 \%)$ & $18(15.8 \%)$ \\
\hline F18:Stx2:Stx2e:EAST-I & 4 & - & 4 \\
\hline AggR & - & 3 & 3 \\
\hline stx1:stx2 & - & 3 & 3 \\
\hline stx1 & - & 2 & 2 \\
\hline F18:Stx2:Stx2e & 2 & - & 2 \\
\hline stx2 & - & 1 & 1 \\
\hline ipaH & - & 1 & 1 \\
\hline F18:Stx2e:EAST-I & 1 & - & 1 \\
\hline _3) & - & 1 & 1 \\
\hline
\end{tabular}

1)* Significant difference between origin of isolates $(p<0.05)$.

${ }^{* *}$ Significant difference between origin of isolates $(p<0.01)$.

2) O_UT: Untypeable

3) Non-virulence gene detected

4) Other serogroup (number of isolates): 06 (3), 018 (3), 0100 (3), 0136 (3), 011 (2), 021 (2), 055 (2), 0146 (2), 015 (1), 028 (1), 057 (1), 078 (1), 091 (1), 098 (1), 0103 (1), 0111 (1), 0121 (1), 0145 (1), $0174(1)$. 


\begin{tabular}{|c|c|c|c|}
\hline \multirow{2}{*}{$\begin{array}{l}\text { O-serogroup } \\
\text { Virotype }\end{array}$} & \multicolumn{3}{|c|}{ No. (\%) of pathogenic E. coli isolates } \\
\hline & Swine $(n=64)$ & Patients $(n=50)$ & Total $(n=114)$ \\
\hline Others ${ }^{4)}$ & $14(21.9 \%)$ & $17(34.0 \%)$ & $31(27.2 \%)$ \\
\hline \multicolumn{4}{|c|}{ 1) * Significant difference between origin of isolates $(p<0.05)$. } \\
\hline \multicolumn{4}{|c|}{${ }^{* *}$ Significant difference between origin of isolates $(p<0.01)$. } \\
\hline \multicolumn{4}{|c|}{ 2) O_UT: Untypeable } \\
\hline \multicolumn{4}{|c|}{ 3) Non-virulence gene detected } \\
\hline \multicolumn{4}{|c|}{$\begin{array}{l}\text { 4) Other serogroup (number of isolates): } 06 \text { (3), } 018 \text { (3), } 0100(3), 0136(3), 011(2), 021(2), 055(2) \text {, } \\
0146(2), 015(1), 028(1), 057(1), 078(1), 091(1), 098(1), 0103(1), 0111(1), 0121(1), 0145(1), \\
0174(1) .\end{array}$} \\
\hline
\end{tabular}

\section{Multi-locus Sequence Typing (Mlst)}

A minimum spanning tree based on MLST data including branch distances is presented in Fig. 1. The divides within each node are equal to the number of isolates belonging to the sequence type it represents. White numbers in the circles indicate the MLST sequence type. Black colored numbers on line indicate the absolute distance between each sequence type. The node sizes vary linearly with the number of isolates of a given sequencing type. The most prevalent ST in swine isolates were ST 1 ( 21 isolates, $32.8 \%)$ and ST 100 (21 isolates, 32.8\%). While swine isolates showed only 10 STs, isolates from patients showed 28 STs. The prevalent STs in isolates from patients were ST 678 (6 isolates, 12.0\%), ST 21 (4 isolates, 8.0\%), and ST 101 (3 isolates, 6.0\%). In both swine and patients' samples, ST 10 (swine: 6 isolates; patients: 2 isolates) and ST 88 (swine: 2 isolates; patients: 1 isolate) were detected simultaneously. Moreover, 5 isolates of swine showed novel STs, ST New. The following STs had 3 absolute distance: ST $34-$ ST 218; ST 34 - ST 10; ST 218 - ST 3744; ST 10 - ST 218; ST New - ST 641; ST 88 - ST 90.

\section{Discussion}

In this study, we compared antimicrobial resistance and virulence genes of $E$. coli isolated from patients suffering from diarrhea and diarrheic pigs. It is noted that the overall prevalence of antimicrobial resistance in the isolates from pigs were higher than in those from humans, which was consistent with a previous study $(14,15)$. This suggests that the use of antimicrobial agents in pig farming may be more frequent than that in humans. Due to the lack of strict regulations on the use of antimicrobials in Korea (16), using antimicrobials indiscriminately by non-specialists, like livestock workers, could increase antimicrobial resistance. Isolates from pigs showed significantly higher resistance to aminoglycosides, fluoroquinolone, tetracyclines, nalidixic acid, trimethoprim / sulfamethoxazole, and chloramphenicol than in isolates from patients. The findings that the highest prevalence of resistance occurred among isolates 
from pigs and that resistance was seen to drug classes approved for use in swine (17) suggest that antimicrobial use in swine may be a factor in the emergence of antimicrobial resistance in $E$. coli. Because these agents are used for treating enteric infections in humans, decreasing resistance to these agents is crucial.

Fluoroquinolones are the primary option for controlling colibacillosis and have been widely used throughout the country for mass medication in pig farms $(17,18)$. The World Health Organization (WHO) and the World Organization for Animal Health (OIE) have classified fluoroquinolones as "critically important antimicrobial agents" because of their importance in both human and animal medicine (19). A previous study in Korea reported high resistance to ciprofloxacin (34.5\%) in Korean pigs (20), which is in accordance with our study. The present study indicated that resistance of $E$. coli to ciprofloxacin (37.5\%) from swine isolates was higher in Korea as compared to that in other countries with advanced swine farming (Netherland: $1.0 \%$, Sweden: $0.0 \%$, US: $0.0 \%)(21,22)$, which restrict the use of antimicrobial agents. This is largely due to the massive use of fluoroquinolone in livestock for therapeutic and selftreatment purposes (quinolone sales: $44,380 \mathrm{~kg}$ ) in Korea. Moreover, the resistance to ciprofloxacin (swine: $37.5 \%$; patients: $10.0 \%$ ) and norfloxacin (swine: $29.7 \%$; patients: $8.0 \%$ ) was higher in swine isolates than in patients' samples. Since fluoroquinolone resistance can be transferred from pigs to humans (23), this becomes a public health hazard, and it is necessary to establish a strategy to reduce antimicrobial resistance. Therefore, additional regulations to reduce the resistance to ciprofloxacin is essential.

Introduced into clinical application in the mid-1990s, aminoglycosides are one class of antimicrobials that are used in veterinary and human medicine and have been used as the primary option for controlling colibacillosis $(17,24)$. In this study, we found higher resistance $(51.6 \%)$ to gentamicin in Korea than in other countries (US: $0.0 \%$; Australia: $7.4 \%)(22,25)$. Gentamicin is no longer used in swine farming in advanced countries (25); however, in Korea, gentamicin is frequently used for the treatment of colibacillosis (5). This difference in the use of antimicrobial agents resulted in high resistance to gentamicin in Korea. Moreover, the resistance to some aminoglycoside antimicrobial agents (gentamicin, kanamycin, and amikacin) was significantly higher in isolates from swine than in those from patients. Due to the adverse events associated with aminoglycosides, such as inner ear toxicity (sensorineural hearing loss) and kidney damage (chronic kidney disease), the use of aminoglycosides is limited and administered for severe infections in humans (26). However, in pigs, aminoglycosides can be used to manage weaning pig scours caused primarily by $E$. coli $(26,27)$. The administration of aminoglycosides in pigs has the potential to generate cross-resistance to vitally important human antimicrobials like amikacin, which is a huge concern for human health (25).

In this study, we found high rates of multidrug resistance (swine: $93.8 \%$; patients: $86.0 \%$ ). Evidently, in our results, values obtained were higher than those obtained in other studies (Pig - Netherland: $34.2 \%(4)$, China: $84.2 \%$ (28), Thailand: 84.6\% (14); Humans - Netherland: 7.1\% (29), China: 15.2\% (28), Thailand: $45.7 \%$ (14)) In Korea, antimicrobial use in veterinary (33.2 defined daily doses (DDD) per 1,000 inhabitants per day) and human medicine (31.7 DDD per 1,000 inhabitants per day) is relatively higher as 
compared to that in other member countries of the Organization for Economic Co-operation and Development (OECD) (21.3 and 23.7 DDD per 1,000 inhabitants per day, respectively) (30, 31). Besides the possible role of increased selective pressure by repeated exposure to therapeutic agents, this is a likely cofactor in the increased frequency of antimicrobial resistance observed among pathogens (32). The high level of antimicrobial resistance is directly linked to challenges in the treatment of diseases; therefore, it is important to manage antimicrobial resistance.

The clinical symptoms of colibacillosis vary depending on the pathotype of infective $E$. coli (33). The most predominant pathotype in Korea was EPEC (34); however, in this study, the most prevalent pathotype in patients was STEC (27 isolates, $54.0 \%$ ), followed by ETEC (11 isolates, $22.0 \%)$. There was no clear reason why the pathotype of $E$. coli had temporal changes, but it is evident that the predominant pathotype has changed to ETEC and STEC from EPEC. STEC is the third most common zoonotic infection within the Europe (32). In this study, 28 isolates (43.8\%) of swine contained STEC. Because STEC is a zoonotic food- and waterborne pathogen of a serious public health concern and it can cause outbreaks, hemorrhagic colitis, and potentially life-threatening complication, such as hemolytic-uremic syndrome $(3,35)$, careful attention should be paid to STEC's pig-human cross-infection.

To produce enterotoxins and cause diseases, pathogenic E. coli needs to first attach to intestines (36). Fimbriae play an important role in allowing $E$. coli to attach to the intestinal mucosa and epithelial cells (37). In late 1990s, the most predominant fimbriae in Korean pigs was F6, which then changed to F5 in the mid-2000s $(38,39)$. However in this study, there was no fimbrial adhesin in isolates from patients, and the most prevalent virotype of $E$. coli in pigs encoded F4 (29 isolates, 45.3\%) and F18 (35 isolates, $54.7 \%$ ). In Korea, inactivated vaccines targeting F4 and F18 are being used nationwide (40). The use of these vaccines could cause the antigenic variations and would account for the prevalence of fimbriae or non-fimbrial adhesins, besides F4 and F18, in pigs.

The stx2 gene was detected both in isolates from pigs (15 isolates, 23.4\%) and patients (19 isolates, $38.0 \%)$. The stx gene was known to be associated with edema disease in swine and hemolytic-uremic syndrome in human $(3,41,42)$. The receptor for stx 2 is globotriosyl ceramide which is seen in both swine and humans (43). Also, the LT and ST gene was detected both in isolates from swine (29 isolates, 45.3\%) and patients (11 isolates, $22.0 \%$ ), which is associated with neonatal or postweaning diarrhea in pigs and in traveler's diarrhea in humans (44). Both enterotoxins elevate the extracellular levels of chloride and bicarbonate ion which could induce osmotic diarrhea (45). There was no common fimbrial adhesin in isolates from both swine and patients. However, several studies reported a high association between nonfimbrial adhesin AIDA-I and F18, which is the most prevalent fimbrial adhesin in the present study (46$48)$, and AIDA-I was detected in pigs (26.6\%), and thus has the ability to cause cross-infection between pigs and humans (16). Furthermore, a recent study indicated that LT also could play a significant role in the enhancement of bacterial adherence (49). Thus, cross-infection of pathogenic $E$. coli between pigs and humans could occur sufficiently. 
There was lower evidence on whether specific 0-serogroup could cause diseases because a limited number of 0 -serogroups have been reported for specific disease $(50,51)$. In this study, the most prevalent 0-serogroup in swine isolates was 0149 (28 isolates, 43.8\%), followed by 0139 (13 isolates, 20.3\%) and 0157 ( 2 isolates, 3.1\%). This is in accordance with the results obtained by Kusumoto et al. (52), which indicated that 0139 was the predominant serogroup in Japan in 2010-2014. Kwon et al. indicated that 0157 and 08 were the predominant O-serogroups in Korea from 1995 to 1997 (53). However, in this study, just two 0157 isolates were detected from pigs. It is apparent that the predominant serogroup has shifted from 0157 to 0149 and 0139 in Korean swine farms. In contrast to swine isolates, 0157 (6 isolates, $12.0 \%$ ) was the predominant serogroup in patients; 0157 is known to be associated with eae, stx 1 and/or stx2 gene (54). Interestingly, swine isolates had no stx2 gene while all isolates from patients encoded stx 1 and/or stx 2 gene. Because of the low number of 0157 strains, it becomes hard to explain the cause of this phenomenon; however, we assumed that the relationship of O-serogroup with virotype has been changing over time.

MLST allows determining the phylogenetic relationships among deep lineages, providing a complementary view of the population structure (55). In this study, we analyzed 64 and 50 E. coli strains from swine and patients, respectively. There were only 10 STs in swine isolates while patients' isolates showed more STs (28 STs). Most strains in pigs were ST 1 (21 isolates, 32.8\%) and ST 100 (21 isolates, $32.8 \%$, indicating that the cause of enteric colibacillosis in pigs had a similar origin. This is accordance with other studies which state that ST 1 and ST 100 isolates are the predominant ETEC type, and are important pig pathogens in the United States, Canada, Germany, and Thailand (http://mlst.warwick.ac.uk $/ \mathrm{mlst} / \mathrm{dbs} /$ Ecoli). In contrast to swine isolates, each ST of isolates from patients had small portions, which means that each strain from patients had been isolated from a different origin. Several studies reported ST 10 as one of the most common strains in human populations, and ST 10 strains commonly carry certain antimicrobial resistance genes (such as ampC-type beta lactamases and NDM-type carbapenemases) $(56,57)$. Also, ST 88 has been previously described in association with c-AmpC production in a French hospital (58). Interestingly, ST 10 (swine: 6 isolates; patients: 3 isolates) and ST 88 (swine 2 isolates; patients: 1 isolate) were detected simultaneously in swine and patients' samples. Additionally, we found that isolates from different hosts (swine and patients) were closely related; ST 34 (patients: 1 isolate) - ST 10 (swine: 6 isolates, patients: 2 isolates), ST 88 (swine: 2 isolates, patients: 1 isolate) - ST 90 (swine: 2 isolates), ST 218 (patients: 3 isolates) - ST 10. In addition, five isolates from swine showing new ST were found that were phylogenetically closed with ST 641. Although, there was a weak relationship between patients' isolates, the emergence of a pathogenic $E$. coli showing new ST may pose not only a problem in veterinary medicine but also a significant public health threat, and therefore is in need of urgent attention (59). We confirmed a phylogenetical association between swine and patients' isolates. Similar ST indicates the risk of emergence of zoonotic disease (60) and a risk for crossinfection, and can even cause antimicrobial resistance to be transferred between pigs and humans in Korea.

\section{Conclusions}


In this study, we analyzed antimicrobial resistance, virulence genes, O-serogroups, and MLST of E. coli from isolates of pigs and patients suffering from diarrhea. Both isolates from swine and patients had the stx2 gene, which could cause severe disease, such as edema disease (swine) and hemorrhagic colitis (human). Isolates from swine showed significantly higher antimicrobial resistance than those from humans, especially in fluoroquinolone and aminoglycosides. Moreover, isolates used in this study showed phylogenetic association. These results provide data that strengthen the understanding of crossinfection and the transfer of antimicrobial resistance through pathogenic $E$. coli between humans and pigs, along with important data that can be used to support the development of vaccines to implement strategies for the control and prevention of antimicrobial resistance.

\section{Declarations}

\section{Ethics approval and consent to participate}

There was no need to approve of ethics because all isolates in this study were already isolated from feces or dead carcasses in Korean swine farm and NCCP.

\section{Consent for publication}

All authors consent to publication of this paper.

\section{Competing interests}

The authors did not provide a conflict of interest statement.

\section{Availability of Data and Materials}

The datasets generated and/or analyzed during the current study are available from the corresponding author on reasonable request.

\section{Funding}

This work was supported by "Korea Institute of Planning and Evaluation for Technology in Food, Agriculture, Forestry and Fisheries (IPET) through Agriculture, Food and Rural Affairs Convergence Technologies Program for Educating Creative Global Leader, funded by Ministry of Agriculture, Food and Rural Affairs (MAFRA) (grant number: 320005-4)".

\section{Authors contribution}

Conception and design of study: Wan-Kyu Lee

Acquisition of data: Kyung-Hyo Do, Kwang-Won Seo

Analysis and/or interpretation of data: Kyung-Hyo Do, Kwang-Won Seo, Wan-Kyu Lee 
Drafting and/or revising the manuscript: Kyung-Hyo Do, Kwang-Won Seo, Wan-Kyu Lee

\section{Acknowledgement}

The pathogen resources from human for this study were provided by the National Culture Collection for Pathogens.

\section{References}

1. RhoumaM,Beaudry F, Thériault, W, Bergeron, N, Laurent-Lewandowski S, Fairbrother JM et al.Impacts of colistin sulfate on fecalEscherichia coli resistance and on growth.Safepork Posters.2015;361-5.

2. DoK-H,ByunJ-W,LeeW-

K.Antimicrobialresistance,adhesinandtoxingenesofporcinepathogenicEscherichia coli followingthebanonantibioticsas thegrowthpromotersinfeed.PakVetJ.2021;41:519-23.

3. JosephA,CointeA,Mariani KurkdjianP,RafatC,HertigA.Shigatoxinassociatedhemolyticuremicsyndrome:Anarrativereview.Toxins(Basel).2020;12(2):146.doi:10.3390/toxins12020067,PMID31973203.

4. European Food Safety Authority,European Centre for Disease Prevention and Control.TheEuropean UnionSummary Report onantimicrobialResistance in zoonotic and indicator bacteria from humans, animals and food in 2018/2019.EFSAJ.2021;19(4):e06490.doi:10.2903/j.efsa.2021.6490,PMID33868492.

5. Animal and PlantQuarantine Agency.Antimicrobial use and antimicrobial resistance monitoring in animals and animal products.Gimcheon,Korea;2019.

6. SzmolkaA,NagyB.Multidrug resistant commensalEscherichia coli in animals and its impact for public health.FrontMicrobiol.2013;4:258.doi:10.3389/fmicb.2013.00258,PMID24027562.

7. IguchiA,lyodaS,SetoK,Morita-IshiharaT,ScheutzF,OhnishiM,Pathogenic E. col/Working Group in Japan.Escherichia coli O-genotypingPCR:Acomprehensive and practical platform for molecular 0 serogrouping.JClinMicrobiol.2015;53(8):2427-32.doi:10.1128/JCM.00321-15,PMID25926488.

8. BaiX,HuB,XuY,SunH,ZhaoA,BaP,et al.Molecular and phylogenetic characterization of non0157ShigaToxin-producingEscherichiacolistrains inChina.FrontCellInfectMicrobiol.2016;6.doi:10.3389/fcimb.2016.00143.

9. DoKH,ByunJW,LeeWK.Prevalence of O-serogroups, virulence genes, and F18 antigenic variants inEscherichia coli isolated from weaned piglets with diarrhea inKoreaduring 20082016.JVetSci.2019;20(1):43-50.doi:10.4142/jvs.2019.20.1.43,PMID30481984.

10. JaureguyF,LandraudL,PassetV,DiancourtL,FrapyE,GuigonG,et al.Phylogenetic and genomic diversity of human bacteremicEscherichia coli strains.BMC Genomics.2008;9:560.doi:10.1186/1471-2164-9560,PMID19036134.

11. Clinical and Laboratory Standards Institute.Performance standards for antimicrobial susceptibility testing;twenty-fourthInformational Supplement, M100-S24;2020. 
12. BauerAW,KirbyWM,SherrisJC,TurckM.Antibiotic susceptibility testing by a standardized single disk method.AmJClinPathol.1966;45(4):493-6.doi:10.1093/ajcp/45.4_ts.493,PMID5325707.

13. MagiorakosA, SrinivasanA,Carey RB, Carmeli Y, Falagas ME, Giske CG, et al.Multidrug-resistant, extensively drug-resistant and pandrug-resistant bacteria:aninternational expert proposal for interim standard definitions for acquired resistance.Microbiology.2011;18:268-81.

14. PungpianC,SinwatN,AngkititrakulS,PrathanR,ChuanchuenR.Presence and Transfer ofantimicrobialResistance Determinants in Escherichia coli in Pigs, Pork, and Humans inThailandand LaoPDRBorder Provinces.Microb Drug Resist.2021;27(4):571-

84.doi:10.1089/mdr.2019.0438,PMID32898454.

15. SinwatN,AngkittitrakulS,CoulsonKF,PilapilFMIR,MeunseneD,ChuanchuenR.High prevalence and molecular characteristics of multidrug-resistantSalmonella in pigs,pork and humans inThailandandLaosprovinces.JMedMicrobiol.2016;65(10):118293.doi:10.1099/jmm.0.000339,PMID27542886.

16. DoKH,ByunJW,LeeWK.Virulence genes and antimicrobial resistance of pathogenicEscherichia coli isolated from diarrheic weaned piglets inKorea.JAnimSciTechnol.2020;62(4):54352.doi:10.5187/jast.2020.62.4.543,PMID32803186.

17. SchroederCM,ZhaoC,DebRoyC,TorcoliniJ,ZhaoS,WhiteDG,et al.Antimicrobialresistanceof Escherichia coli 0157isolatedfromhumans,cattle,swine,andfood.ApplEnvironMicrobiol.2002;68(2):57681.doi:10.1128/AEM.68.2.576-581.2002,PMID11823193.

18. DaviesR,WalesA.Antimicrobialresistanceonfarms:areviewincludingbiosecurity and thepotentialroleofdisinfectantsinresistanceselection.ComprRev Food Sci Food Saf.2019;18(3):75374.doi:10.1111/1541-4337.12438,PMID33336931.

19. FAO.Joint FAO/WHO/OIE expert meeting on critically important antimicrobials. In:Proceedings of the FAO/WHO/OIEexpertmeetingFAOheadquarters.

20. HuYS,ShinS,ParkYH,ParkKT.Prevalence andmechanismoffluoroquinoloneresistanceinEscherichia coli isolatedfromswinefecesinKorea.J Food Prot.2017;80(7):1145-51.doi:10.4315/0362-028X.JFP-16502,PMID28598203.

21. Van Den BogaardAEJM,LondonN,StobberinghEE.Antimicrobial resistance in pig faecal samples fromtheNetherlands(five abattoirs) andSweden.JAntimicrobChemother.2000;45(5):66371.doi:10.1093/jac/45.5.663,PMID10797090.

22. SayahRS,KaneeneJB,JohnsonY,MillerR.Patterns ofantimicrobialresistanceobservedinEscherichia coli isolatesobtainedfromdomestic- andwild-animalfecalsamples, humanSeptage, andsurfacewater.ApplEnvironMicrobiol.2005;71(3):1394-404.doi:10.1128/AEM.71.3.13941404.2005,PMID15746342.

23. BartonMD.Impact of antibiotic use in the swine industry.CurrOpinMicrobiol.2014;19:915.doi:10.1016/j.mib.2014.05.017,PMID24959754.

24. ZarrilliR,TripodiMF,Di PopoloA,FortunatoR,BagattiniM,CrispinoM,et al.Molecular epidemiology of high-levelaminoglycoside-resistant enterococci isolated from patients in a university hospital in 
southernItaly.JAntimicrobChemother.2005;56(5):827-35.doi:10.1093/jac/dki347,PMID16186168.

25. van BredaLK,DhungyelOP,WardMP.Antibiotic resistantEscherichia coli in southeastern Australian pig herds and implications for surveillance.Zoonoses Public Health.2018;65(1):e1-

7.doi:10.1111/zph.12402,PMID28921905.

26. JacksonCR,Fedorka-CrayPJ,BarrettJB,LadelySR.High-levelaminoglycosideresistant enterococci isolated from swine.Epidemiollnfect.2005;133(2):367-

71.doi:10.1017/s0950268804003395,PMID15816164.

27. AarestrupFM,Oliver DuranC,BurchDGS.Antimicrobial resistance in swine production.Anim Health ResRev.2008;9(2):135-48.doi:10.1017/S1466252308001503,PMID18983723.

28. PanY,HuB,BaiX,YangX,CaoL,LiuQ,et al.Antimicrobial resistance of non-0157 shiga toxinproducingEscherichia coli isolated from humans and domestic animals.Antibiotics(Basel).2021;10(1):1-13.doi:10.3390/antibiotics10010074,PMID33466678.

29. JohnsonTJ,LogueCM,JohnsonJR,KuskowskiMA,SherwoodJS,BarnesHJ,et al.Associations between multidrug resistance, plasmid content, and virulence potential among extraintestinal pathogenic and commensalEscherichia coli from humans and poultry.FoodbornePathogDis.2012;9(1):3746.doi:10.1089/fpd.2011.0961,PMID21988401.

30. Korea Institute for Health and Social Affairs.Drug use evaluation.Seoul,South Korea:Korea Institute for Health and Social Affairs;2000.

31. Organization for Economic Co-operation and Development.Antimicrobial resistance.OECD.Available from:http://www.oecd.org/els/health-systems/antimicrobial-resistance.htm.

32. BoerlinP,McEwenSA,Boerlin-PetzoldF,WilsonJB,JohnsonRP,GylesCL.Associations between virulence factors ofShigatoxin-producingEscherichia coli and disease in humans.JClinMicrobiol.1999;37(3):497-503.doi:10.1128/JCM.37.3.497-503.1999,PMID9986802.

33. PaulN.Review Virulence nature of Escherichia coli in neonatal swine.OnlineJ.Anim.FeedRes.2015;5:169-74.

34. LeeJH,ChoHT,KimYH,KangHJ,ChalH.Isolation of enteropathogenicEscherichia coli, thermophilicCampylobacterand Salmonelleae from scouring piglets.KoreanJVetRes(KoreaR.)28.1988:67-73.

35. BrilhanteM,PerretenV,DonàV.Multidrug resistance and multivirulence plasmids in enterotoxigenic and hybridShigatoxin-producing/enterotoxigenicEscherichia coli isolated from diarrheic pigs inSwitzerland.VetJ.2019;244:60-8.doi:10.1016/j.tvjl.2018.12.015,PMID30825896.

36. DoK-H,ByunJ-W,LeeW-K.Serogroups,virulencegenesandantimicrobialresistanceof F4+ and F18+Escherichia coli isolatedfromweanedpiglets.PakVetJ.2019;39(2):26670.doi:10.29261/pakvetj/2019.021.

37. DuanQ,NandreR,ZhouM,ZhuG. Type I fimbriae mediate in vitro adherence of porcine F18ac+enterotoxigenicEscherichia coli (ETEC).AnnMicrobiol.2017;67(12):793-9.doi:10.1007/s13213017-1305-z. 
38. KwonD,ChoiC,JungT,ChungHK,KimJP,BaeSS,et al.Genotypic prevalence of the fimbrial adhesins (F4, F5, F6, F41 and F18) and toxins (LT,STa,STband Sbc2e) in Escherichia coli isolated from postweaning pigs with diarrhoea or oedema disease inKorea.VetRec.2002;150(2):357.doi:10.1136/vr.150.2.35,PMID11829064.

39. LeeSI,RayamahjiN,LeeWJ,ChaSB,ShinMK,RohYM,et al.Genotypes, antibiogram, and pulsed-field gel electrophoresis profiles of Escherichia coli strains from piglets inKorea.JVetDiagnInvest.2009;21(4):510-6.doi:10.1177/104063870902100413,PMID19564500.

40. DoKH,ParkHE,ByunJW,LeeWK.Virulence and antimicrobial resistance profiles ofEscherichia coliencoding mcr gene from diarrhoeic weaned piglets inKoreaduring 2007-2016.J Glob AntimicrobResist.2020;20:324-7.doi:10.1016/j.jgar.2019.09.010,PMID31542552.

41. JungJ,KimH,JoA,KimJ,LeeW,ByunJ.Enrichment media for Stx2e production inShigatoxinproducingEscherichia coli.JBiomedTransIRes.2017;18(3):103-7.doi:10.12729/jbtr.2017.18.3.103.

42. LeeJB,HanD,LeeHT,WiSM,ParkJH,JoJW,et al.Pathogenic and phylogenetic characteristics of non0157Shigatoxin-producing Escherichiacoliisolates from retail meats inSouth Korea.JVetSci.2018;19(2):251-9.doi:10.4142/jvs.2018.19.2.251,PMID29284205.

43. WaddellTE,LingwoodCA,GylesCL.Interaction of verotoxin2èmewith pig intestine.InfectImmun.1996;64(5):1714-9.doi:10.1128/iai.64.5.1714-1719.1996,PMID8613382.

44. NeutC.Carriage of multidrug-resistant bacteria in healthy people:recognition of several risk groups.Antibiotics(Basel).2021;10(10).doi:10.3390/antibiotics10101163,PMID34680744.

45. LuppiA.Swine enteric colibacillosis:diagnosis, therapy and antimicrobial resistance.Porc.Porcine Health Manag.2017;3:16.doi:10.1186/s40813-017-0063-4,PMID28794894.

46. NiewerthU,FreyA,VossT,Le BouguénecC,BaljerG,FrankeS,et al.TheAIDAautotransporterSystem Is Associated with F18 and Stx2e inEscherichiaco/lsolates from Pigs Diagnosed withedemaDisease and Postweaningdiarrhea.ClinDiagnLabImmunol.2001;8(1):143-9.doi:10.1128/CDLI.8.1.143149.2001,PMID11139209.

47. ZhangW,ZhaoM,RueschL,OmotA,FrancisD.Prevalence of virulence genes inEscherichia coli strains recently isolated from young pigs with diarrhea in the US.VetMicrobiol.2007;123(1-3):14552.doi:10.1016/j.vetmic.2007.02.018,PMID17368762.

48. ZhaoL,ChenX,XuX,SongG,LiuX.Analysis of theAIDA-Igene sequence and prevalence in Escherichia coli isolates from pigs with post-weaning diarrhoea and oedema disease.VetJ.2009;180(1):1249.doi:10.1016/j.tvjl.2007.10.021,PMID18077196.

49. DuanQ,XiaP,NandreR,ZhangW,ZhuG.Review ofnewlyidentifiedfunctionsassociatedwiththeheatlabiletoxinofenterotoxigenicEscherichia coli.FrontCellInfectMicrobiol.2019;9:292.doi:10.3389/fcimb.2019.00292,PMID31456954.

50. VilaJ,Sáez-López E,Johnson JR,Römling U,Dobrindt U,Cantón R, et al.Escherichia coli:anold friend with new tidings.FEMSMicrobiol. Rev. fuw005.2016;40(4):437-

63.doi:10.1093/femsre/fuw005,PMID28201713. 
51. ImberechtsH,De GreveH,LintermansP.The pathogenesis of edema disease in pigs. A review.VetMicrobiol.1992;31(2-3):221-33.doi:10.1016/0378-1135(92)90080-d,PMID1626371.

52. KusumotoM,HikodaY,FujiiY,MurataM,MiyoshiH,OguraY,et al.Emergence of amultidrugresistantShigatoxin-producingenterotoxigenicEscherichiacoliLineage indiseasedswineinJapan.JClinMicrobiol.2016;54(4):1074-81.doi:10.1128/JCM.0314115,PMID26865687.

53. KwonD,Kim0,ChaeC.Prevalence of genotypes for fimbriae and enterotoxins and of $\mathrm{O}$ serogroups inEscherichia coli isolated from diarrheic piglets inKorea.J Vet Diagn Invest.1999;11(2):14651.doi:10.1177/104063879901100207,PMID10098686.

54. StrawBE,ZimmermanJJ,D'AllaireS,TaylorDJ.Diseases of swine.9thed;2006. p.387-95.

55. ChattawayMA,DayM,MtwaleJ,WhiteE,RogersJ,DayM,et al.Clonality, virulence and antimicrobial resistance of enteroaggregativeEscherichiacolifrom Mirzapur,Bangladesh.JMedMicrobiol.2017;66(10):142935.doi:10.1099/jmm.0.000594,PMID28945190.

56. YuF,ChenX,ZhengS,HanD,WangY,WangR,et al.Prevalence and genetic diversity of human diarrheagenicEscherichia coli isolates by multilocus sequence typing.IntJInfectDis.2018;67:713.doi:10.1016/j.ijid.2017.11.025,PMID29183841.

57. SavinM,BierbaumG,KreyenschmidtJ,SchmithausenRM,SibE,SchmogerS,et al.Clinically relevantEscherichia coli isolates from process waters and wastewater of poultry and pig slaughterhouses in germany.Microorganisms.2021;9(4):117.doi:10.3390/microorganisms9040698,PMID33800539.

58. CrémetL,CaroffN,GiraudeauC,DauvergneS,LepelletierD,ReynaudA,et al.Occurrence of ST23 complex phylogroup aEscherichia coli isolates producing extended-spectrum AmpC $\beta$-lactamase in aFrenchhospital.Antimicrob Agents Chemother.2010;54(5):2216-8.doi:10.1128/AAC.0158009,PMID20145079.

59. Nicolas-ChanoineMH,BlancoJ,Leflon-GuiboutV,DemartyR,AlonsoMP,CaniçaMM,et al.Intercontinental emergence of Escherichia coliclone 025:H4-ST131 producing CTX-M15.JAntimicrobChemother.2008;61(2):273-81.doi:10.1093/jac/dkm464,PMID18077311.

60. YangC,ShaoW,WeiL,ChenL,ZhuA,PanZ.SubtypingSalmonellaisolated from pet dogs withmultilocus sequence typing(MLST)andclustered regularly interspaced short palindromic repeats(CRISPRs).AMB Express.2021;11(1):60.doi:10.1186/s13568-021-01221-9,PMID33893895.

\section{Figures}

\section{Figure 1}

Minimum spanning tree based on sequence type of E. coli from pigs and humans 